\title{
Improvements in Unmarried African American Parents' Rapport, Communication, and Problem-Solving Following a Prenatal Coparenting Intervention
}

\author{
JAMES P. MCHALE* \\ SELIN SALMAN-ENGIN ${ }^{\dagger}$ \\ MICHAEL D. COOVERT
}

This report examines effects of a coparenting intervention designed for and delivered to expectant unmarried African American mothers and fathers on observed interaction dynamics known to predict relationship adjustment. Twenty families took part in the sixsession "Figuring It Out for the Child" (FIOC) dyadic intervention offered in a faith-based human services agency during the third trimester of the mother's pregnancy, and completed a postpartum booster session 1 month after the baby's arrival. Parent referrals for the FIOC program were received from a county Health Department and from OBGYNs and Pregnancy Centers in the targeted community. All intervention sessions were delivered by a trained male-female paraprofessional team whose fidelity to the FIOC manualized curriculum was independently evaluated by a team of trained analysts. At both the point of intake ("PRE") and again at an exit evaluation completed 3 months postpartum ("POST"), the mothers and fathers were videotaped as they completed two standardized "revealed differences" conflict discussions. Blinded videotapes of these sessions were evaluated using the System for Coding Interactions in Dyads. Analyses documented statistically significant improvements on 8 of 12 variables examined, with effect sizes ranging from moderate to large. Overall, 14 families demonstrated beneficial outcomes, 3 did not improve, and 3 showed some signs of decline from the point of intake. For most interaction processes, PRE to POST improvements were unrelated to degree of adherence the paraprofessional interventionists showed to the curriculum. However, better interventionist competence was related to decreases in partners' Coerciveness and Negativity and Conflict, and to smaller increases in partner Withdrawal. Implications of the work for development and delivery of community-based coparenting interventions for unmarried parents are discussed.

*University of South Florida St. Petersburg, St. Petersburg, FL.

${ }^{\dagger}$ Psychology Department, Bilkent University, Ankara, Turkey.

tUniversity of South Florida, Tampa, FL.

Correspondence concerning this article should be addressed to James P. McHale, Director, Family Study Center, University of South Florida St. Petersburg, 140 Seventh Avenue South, St. Petersburg, FL 33701; E-mail: jmchale@mail.usf.edu.

Work on this project was supported by a grant from the Brady Education Foundation. We thank Mt. Zion Human Services and the Pinellas County Health Department for their partnership on this project; Mari Kittle, Jessica Gordon, Rashid Mizell, Eric Armstrong, and Florence Guillett for their efforts in recruitment, family interviews, and assessments; Kyle DePalma for database management and support; the interventionists, supervisors, and Family Study Center staff who helped participant families feel welcome and valued; and above all, the families who provided a deeply personal glimpse into their lives. This work, and the impact it stands to make in our field, would have been impossible without them and their commitment to and vision for their children. No author has any conflict of interest related to this article. 
Keywords: Prenatal intervention; Coparenting; Fragile families; Rapport; Problem-solving; Communication; African American fathers

Fam Proc 54:619-629, 2015

\section{INTRODUCTION}

$\mathrm{D}$ espite the fact that the majority of families headed by unmarried parents will never transition to marriage, very little national attention has been devoted to developing interventions targeting establishment of positive and lasting coparenting alliances in such families (McHale, Waller, \& Pearson, 2012). Though recent years have seen multiple commentaries, reviews, and meta-analyses of Marriage and Relationship Enhancement (MRE) programs - and a thoughtful processing of results from the federally funded Building Strong Families (BSF) MRE initiative designed to serve low-income unmarried, romantically involved parents who were expecting or who had recently had a baby-the focus of such reviews has remained on dyadic couple rather than triangular coparenting relationships. BSF programming focused principally on couple relationship enhancement with comparatively little attention given to promoting coparenting in the context of noncommitted partnerships. This knowledge gap is a significant one because positive coparenting alliances formed between adults during infancy and early childhood-regardless of whether or not those individuals are heterosexual, married biological parents - can confer substantial benefit to children (Minuchin, 1974; Minuchin, Colapinto, \& Minuchin, 2009). Mothers report greater parenting efficacy, fathers show more positive engagement, and infants and toddlers demonstrate better socioemotional development (see McHale \& Lindahl, 2011, for a comprehensive review of this literature).

In this report, we present results from a pilot field test of a new coparenting intervention designed specifically for unmarried African American parents having their first child together. In conjunction with other reports in this special issue, this community-based intervention is among the first to deliver an intervention for unmarried parents where coparenting is the prime focus, and where the focal change measure of program benefit relies on observations of mother-father interaction process pre- and several months postintervention, rather than relying on self-reports from program participants as have the preponderance of all evaluations of MRE programs.

\section{BACKGROUND}

The U.S. federal government has invested in voluntary MRE programs as one strategy to augment existing policy efforts to strengthen families, promote child wellbeing, and reduce poverty. One flagship program has been the large, well-endowed BSF study for low-income unmarried, romantically involved pregnant or parenting mothers and fathers. Yet BSF's focus on committed, romantically involved relationships did not always meet unmarried mothers and fathers "where they are"; in fact, BSF eligibility criteria actually disqualified many higher risk unmarried families from participation. As one illustration, according to a 2006 report by Dion and colleagues describing early recruitment efforts at two program sites where BSF recruitment was combined with the recruitment process for the host Healthy Families program (which serves pregnant or new mothers at risk for child abuse), fewer than $10 \%$ of families met BSF eligibility criteria. One site screening 3,735 individuals for Healthy Families identified and received informed consent from only 117 couples meeting BSF eligibility criteria. The second assessed 1,559 individuals to obtain 143 BSF-eligible couples. The experiences of these sites reflect the reality that 
many higher risk families in the overall Healthy Families target population fell outside BSF's target population. That is, in many families, the parents did not have a romantic relationship or the two parents did not maintain regular contact with one another.

Perhaps more unexpectedly, preliminary evaluations of BSF documented no overall beneficial effects of the intervention for relationship stability or improved coparenting, and further indicated that only about $10 \%$ of recruited-in couples had actually stayed involved long enough to receive a strong dosage of the curriculum (Wood, McConnell, Moore, Clarkwest, \& Hsueh, 2010). Moreover, "treatment-on-the-treated" or TOT analyses augmenting the intent-to-treat or ITT analyses (Wood, Moore, \& Clarkwest, 2011) generally replicated the ITT analyses, suggesting that higher dosages of the program would not have produced significant results for the treatment-group participants. Perhaps of greatest concern were findings that one of the eight main project sites actually showed deleterious effects of the intervention (more relationship break-ups, poorer coparenting, and more reports of domestic violence in BSF couples than in the control group), raising significant questions about the benefits of MRE, at least as conceived for the BSF project, for higher risk low-income couples. At the same time, analyses indicated several more hopeful findings. One of the eight sites did demonstrate positive benefits and that site has understandably been the target of intensive scrutiny in search of lessons learned and growing points for MRE efforts (Devaney \& Dion, 2010).

Also of consequence, committed African American couples who participated in BSF were found to be more likely than other sub-groups to have derived positive benefit from the programming (more faithful to one another, less likely to experience abuse, more cooperation in coparenting). Evidence from another recent report likewise indicates that unmarried but committed African American coparents may derive benefit from targeted interventions. Owen, Quirk, Bergen, Inch, and France (2012) found some benefits of the Prevention and Relationship Enhancement Program/PREP program, which was not one of the three RME curricula chosen for the BSF project, for lower income African American and Latino couples. Of note, they also found that couples who participated in PREP's dyadic format did better than those in PREP's group format. These findings suggest that under the right circumstances, RME interventions may hold interest and utility for unmarried African American families, at least those in more committed relationships.

For other unmarried families, alternative models and approaches may be worth investigating. Many commentators (e.g., Edin \& Reed, 2005; Huston \& Melz, 2004; Karney, 2011) have questioned the thrust of RME interventions seeking to convince poor Americans of the value of marriage, advocating that interventions instead focus on harmful effects of high-conflict parental relationships and on benefits to children of harmonious coparental functioning (Edin \& Reed, 2005). Programs would also do well, they argued, to "meet families where they are" and improve unmarried parents' interparental relationship quality by tackling real-life challenges, such as presence of children from prior unions and financial instability.

\section{SUMMARY AND PROSPECTUS}

This study addressed a major gap in the family-strengthening literature, evaluating an insight- and skills-based Focused Coparenting Consultation (McHale \& Irace, 2010) delivered in a dyadic format to unmarried African American men and women expecting a first child together. The "Figuring It Out for the Child" (FIOC; McHale, Gaskin-Butler, McKay, \& Gallardo, 2013) intervention aimed to heighten awareness about the beneficial impact of positive coparenting for young children's adaptation and mental health, to enhance rapport and solidarity, and to help parents develop the communication and problem-solving skills they will need to surmount the challenges they face in developing a positive and 
sustained coparenting alliance, in or outside of committed cohabitation and/or marriage. To examine whether FIOC had the desired impacts, we assessed and reported on observed changes in mother-father interpersonal dynamics during conflict discussions-dynamics that had initially been assessed at the point of prenatal intake-3-4 months after completion of the FIOC intervention. We also examine whether pre-to-post changes were greater for coparents who came to the intervention having experienced greater accumulated risk and/or for coparents who took part in interventions in which Mentors displayed greater fidelity (adherence and competence) in delivering the FIOC intervention as intended.

\section{METHOD}

\section{Participants}

Twenty unmarried African American families are the focus of this report. Mothers $(M=21.85, S D=4.79$, range $=14-31)$ were slightly younger than fathers $(M=$ 23.45, $S D=5.74$, range $=14-40) ; 4$ mothers and 2 fathers were under 18 at the point of intake. Earnings of all study participants were $200 \%$ or more below the poverty line. Though this was the first baby together for all 40 study participants, five mothers and five fathers had children from prior relationships. In 100\% of the families, the father was present at the time of the baby's birth, and had his name placed on the child's birth certificate.

\section{Recruitment}

Fifty-six mothers were referred by Health Department Healthy Families staff, faithbased organizations, and area pregnancy centers and OBGYNs, with eligibility based on (a) parents being unmarried; (b) the baby being the first for the two parents together (though one or both parents could have children from prior unions); (c) the baby's father having expressed interest in being involved during the pregnancy; and (d) there being no known intimate partner violence (IPV) concerns. Of the 56 referred women, 34 (61\%) expressed interest in participating; two of the 34 were ineligible due to IPV. Successful recruitment of father was accomplished in $88 \%$ (28 of 32) cases where mothers were interested. Twenty of the 28 consented families (71\%) fully completed the six-session intervention and 7 th (booster) session.

\section{Procedure}

Each family was assessed twice. Intake evaluations during the pregnancy's third trimester were at a faith-based community human services agency where the FIOC intervention was delivered. Trained research assistants explained the study, obtained consent, administered assessments, and then introduced the parents to the male-female mentor team with whom they would be working to complete the FIOC curriculum. The second evaluation at a 3-months postpartum exit interview was completed either in the same faith-based agency or, if scheduling challenges dictated, in a university-based Family Study Center. The exit evaluation protocol mirrored that of the intake session. Each parent first completed a personal interview protocol, which included the risk items described below, and then took part in a revealed difference dyadic interaction task in which they discussed two areas of unresolved difference between them-one issue identified by the father in his interview with the male research assistant, and the other identified by the mother during her interview with the female research assistant. The format of this task has been outlined in detail elsewhere (McHale, 2007). Each of the revealed difference discussions were videotaped and later rated by trained multi-ethnic coders experienced with the System for Coding Interactions in Dyads (SCID; Malik \& Lindahl, 2004). 
All FIOC intervention sessions were audiotaped. Blind, trained raters evaluated fidelity of Mentors to the curriculum. Coders independently assessed fidelity from audiotapes and verbatim transcripts of the sessions, using FIOC-specific accomplishment forms developed for each session. Forms and their items, session dependent as each session called for unique activities to be accomplished, enumerated all key activities to be accomplished in each session. Raters coded how competently and completely mentors assisted parents in accomplishing each required activity. After independently rating sessions, coders conferred to debrief discrepancies between their scores. Consensus scores were used as the final data points for each case.

\section{Measures}

\section{Cumulative risk}

Risk was estimated per-family from a set of eight items drawn from the Fragile Families and Child Wellbeing baseline interview protocol. On six indicators, a score of 0 (risk not present for either parent) or 1 (risk present for at least one parent) was given. These items were: presence of children from other unions $(M=.35, S D=.49)$; history of trauma or abuse $(M=.65, S D=.49)$; history of substance abuse $(M=.10, S D=.31)$; history of mental health problems $(M=.35, S D=.49)$; past incarceration $(M=.60, S D=.51)$; and living with at least one biological parent at age $15(0=$ Yes; $1=$ No; $M=1.00, S D=0)$. The two other indicators were continuous: (poor) relationship status (from 0-romantic involvement on a steady basis, to 4 -parents never talking to one another; $M=.20$, $S D=.52$ ); and (absence of) kin support (from 0-very close to family, to 4-no regular contact with family members, $M=1.50, S D=1.10$ ). The eight items were converted to z scores and then summed to create a cumulative family risk index.

\section{Coparents' interaction dynamics during problem-solving discussions}

Malik and Lindahl's (2004) SCID was used to evaluate coparents' rapport, communication, and problem-solving skill. The SCID behaviorally assesses global aspects of affective and communicative functioning in marital and nonmarried dyads. It assesses both theoretically and empirically derived aspects of couple functioning such as communication skills and processes and issues related to the balance of power, dominance, and aggression in relationships. The SCID is reliable across a variety of ethnic groups, including African American couples. Nine codes are rated separately for mothers and fathers using a Likert scale ranging from 1 (Very Low) to 5 (High); mother and father individual ratings were combined for each of the nine individual codes, yielding an effective score range from 2-10. Three codes (Negative Escalation, Cohesiveness, and Pursuit-Withdrawal) are rated for the couple on a 1-5 scale; and two codes, Conflict Management Style and Balance of Power, are categorical. Brief descriptions of all codes follow:

Verbal aggression assesses degree of usage of hostile and aggressive remarks.

Coerciveness assesses degree of usage of threatening statements, gestures, or voice tone.

Attempts to Control are direct nonthreatening statements intended to alter partner thoughts, feelings, and/or actions.

Negativity \& Conflict assesses the level of tension, irritation, and anger that are not demeaning or aggressive.

Withdrawal assesses the degree of removal from or avoidance of the interaction or discussion, witnessed through body language, tone of voice, and/or attitude.

Dysphoric Affect is an emotional/behavioral code assessing overall sadness, sorrow, anguish, grief, pain, regret, hopelessness, disappointment, despair, discouragement, and remorse. 
Problem-Solving Communication assesses the degree to which partners are able to discuss their own feelings and opinions in a constructive manner, and the extent to which partner behaviors facilitate or promote the problem-solving discussion.

Support assesses the degree to which the partner is supportive of and attuned to the other partner by listening carefully, being sensitive to the emotions and concerns of the other, and validating and attempting to understand the other partner's perspective.

Positive Affect measures positive tone of voice, facial expression, and body language.

Negative escalation evaluates how often negative behaviors of one partner are responded to with negative behaviors from the other partner, creating an escalating cycle.

Cohesiveness represents the sense of unity, closeness, and teamwork with a couple.

Pursuit/Withdrawal occurs when one partner's press to discuss an issue or to change is met by attempts to avoid discussion by withdrawing, changing topic, or not responding.

Conflict Management Style assesses the style of couples as either (fe)male pursuit/ (fe)male withdrawal, disengaged, high conflict-hostile, high conflict-expressive, or harmonious.

Balance of power assesses the distribution of power within the couple with four categories: balanced, male dominant, female dominant, conflictual imbalance.

\section{Fidelity of Mentors to the curriculum}

Two dimensions of fidelity were assessed: Competence and Adherence (Salman-Engin et al., 2013). Competence captured skillfulness in performance of Mentors, and Adherence how well the Mentors followed the program curriculum. Number of per-session Adherence items ranged from 4 to 10 . Raters gave a score of " 0 " if they judged that a specific deliverable had not been attained. They gave a score of " 1 " if the deliverable was partially accomplished and a rating of " 2 " if the item was satisfactorily accomplished as intended. To assess Competence, two different scales were adapted and combined. On both, coders rated male and female mentors separately for each session using a 9-point scale $(1-3=$ needs work; 4-6 = acceptable; 7-9 = good work). Sixteen items were drawn from the Fidelity Checklist of Breitenstein et al. (2010), and four items (knowledge, structure, process skills, and overall quality) from the Fidelity of Implementation Rating System of Knutson, Forgatch, Rains, and Sigmarsdóttir (2009). Normalized scores for Adherence and Competence were summed across all 7 sessions for each family. An average of scores received by both male and female mentors was calculated, yielding one Competence and one Adherence score per participating couple. A Fidelity variable was also computed by combining (adding) normalized Competence and Adherence scores.

\section{RESULTS}

\section{Status of couples at the point of intake}

During the prenatal intake evaluation, 7 of the 20 mother-father pairs were judged to be showing High Conflict-Hostile (6) or High Conflict-Expressive (1) dynamics, with the conflict between the former six sets of coparents presenting as concerning and destructive; the disputes were obvious, intense, tended to escalate and to be mean spirited, and were poorly resolved or not resolved at all. Another four mother-father pairs were judged as either Disengaged (three families) or characterized by a Pursuit-Withdrawal dynamic (one family), indicating that one or both partners showed passivity, disinterest, detachment, or positioned to avoid engaging in the interaction. Discussions were characterized by awkwardness and evasiveness with few to no sustained efforts to engage in discussing the assigned topics. The remaining nine mother-father pairs (45\%) were judged Harmonious on the basis of their interactions at intake, signifying that the dominant impression 
from their discussion was one of connection and warmth between coparents. The level of conflict for these coparents varied from low to high, but quality of the conflict was never destructive. In addition, disputes generally were diffused by the end of the discussion.

\section{Changes in mother-father dynamics from PRE to POST evaluation periods}

Table 1 summarizes mean scores for the 12 continuous variables at PRE and POST assessment.

Paired $t$-tests examining pre-to-post change indicated that four of eight negative coparent dynamics had lessened significantly by 3 months postpartum, 3-4 months after the FIOC intervention. A fifth (Negative Escalation) had also lessened and was significant at a trend level. Only Dysphoric Affect and Pursuit-Withdrawal, which did not show significant changes, and Withdrawal, which showed a trend toward increase, did not witness positive gains (Figure 1).

Paired $t$-tests also indicated that mother-father communication and rapport improved over time; all four positively valenced variables showed significant positive gains following the FIOC intervention and birth of the baby (see Figure 2). Moreover, effect sizes for variables showing statistically significant changes or trends toward significant changes in couple process over time were all moderate to large. Individual effect sizes (Cohen's $d$ ) were as follows: Verbal Aggression: .67; Coerciveness: .67; Attempts to Control: .66; Negativity and Conflict: .77; Withdrawal: .30; Negative Escalation: .53; Problem-Solving Communication: .63; Support: .60; Positive Affect: .67; and—most notably-Cohesiveness: .99.

\section{Was greater Mentor fidelity in delivering the curriculum associated with more positive change in coparents' interactions?}

Bivariate correlational analyses revealed that Mentor Competence (but not Adherence) was significantly related to declines in Coerciveness $(r=.80, p<.001)$ and also to Negativity and Conflict at a trend level $(r=.40, p<.10)$. There was also a trend linking better Mentor Competence $(r=-.43, p<.10)$ and Adherence $(r=-.44, p<.10)$ to smaller increases in Withdrawal. That is, though overall, parents in the study tended to show more withdrawal at POST assessment than at PRE, the degree of increase in postintervention levels of withdrawal was less if mentors showed greater Competence and Adherence in intervention. The combined Fidelity score was also positively related to a

TABLE 1

Descriptive Data for Continuous Mother-Father Interaction Variables at PRE and POST

\begin{tabular}{|c|c|c|c|c|c|c|}
\hline & \multicolumn{3}{|c|}{ Pre } & \multicolumn{3}{|c|}{ Post } \\
\hline & $M$ & $S D$ & Range & $M$ & $S D$ & Range \\
\hline Verbal Aggression & 4.18 & 1.76 & $2-8$ & 3.00 & 1.45 & $2-7$ \\
\hline Coerciveness & 3.59 & 1.82 & $2-9$ & 2.55 & 1.05 & $2-6$ \\
\hline Attempts to Control & 4.86 & 2.12 & $2-9$ & 3.50 & 1.67 & $2-8$ \\
\hline Negativity \& Conflict & 5.64 & 2.40 & $2-10$ & 3.70 & 1.59 & $2-7$ \\
\hline Withdrawal & 3.82 & 1.82 & $2-8$ & 4.50 & 1.73 & $2-7$ \\
\hline Dysphoric Affect & 2.91 & 1.19 & $2-6$ & 2.55 & 0.83 & $2-5$ \\
\hline Problem-Solving Communication & 4.59 & 1.56 & $2-8$ & 6.25 & 2.40 & $2-10$ \\
\hline Support & 4.32 & 2.08 & $2-8$ & 6.10 & 2.55 & $2-10$ \\
\hline Positive Affect & 4.32 & 2.28 & $2-8$ & 6.20 & 2.73 & $2-10$ \\
\hline Negative Escalation & 2.14 & 1.17 & $1-5$ & 1.40 & 0.60 & $1-3$ \\
\hline Cohesiveness & 2.27 & 1.28 & $1-5$ & 3.55 & 1.47 & $1-5$ \\
\hline Pursuit-Withdrawal & 1.46 & 0.74 & $1-4$ & 1.55 & 0.69 & $1-3$ \\
\hline
\end{tabular}

Fam. Proc., Vol. 54, December, 2015 


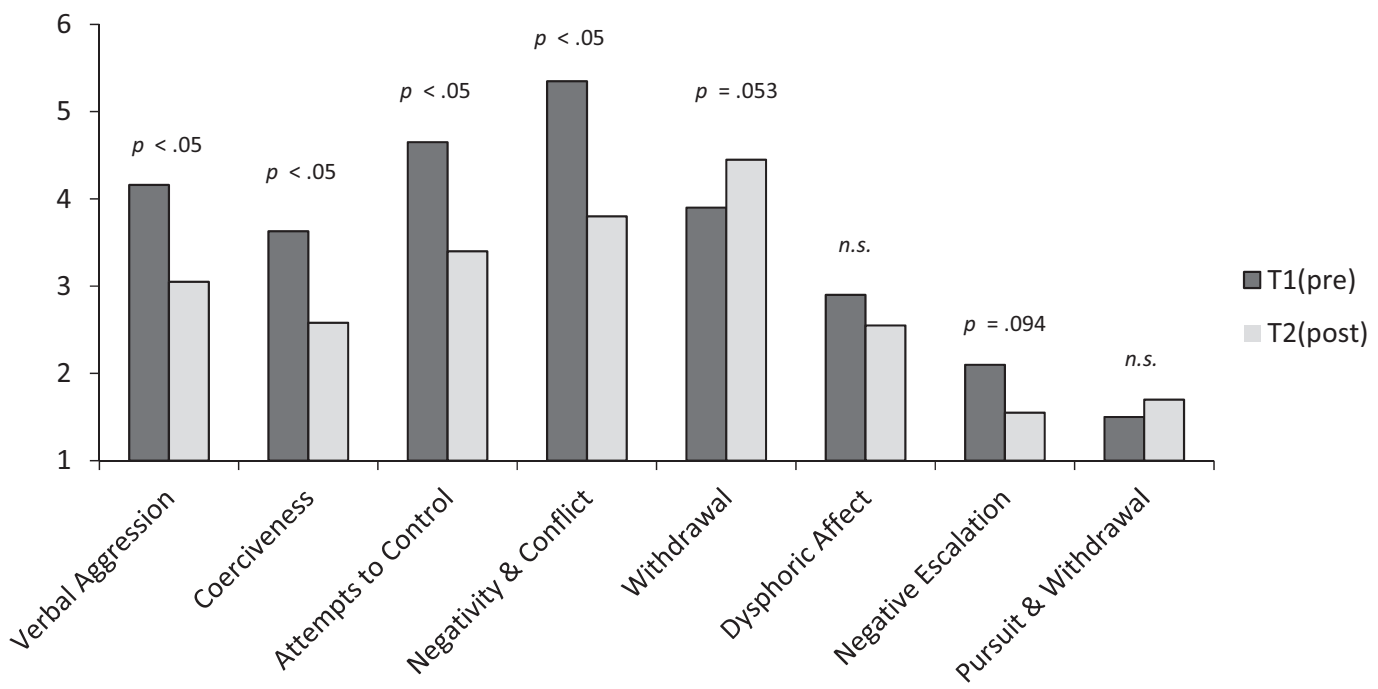

FigURE 1. Mean scores for negative couple interaction variables. $p$ values represent the significance of paired $t$-tests comparing T1 (PRE) with T2 (POST).

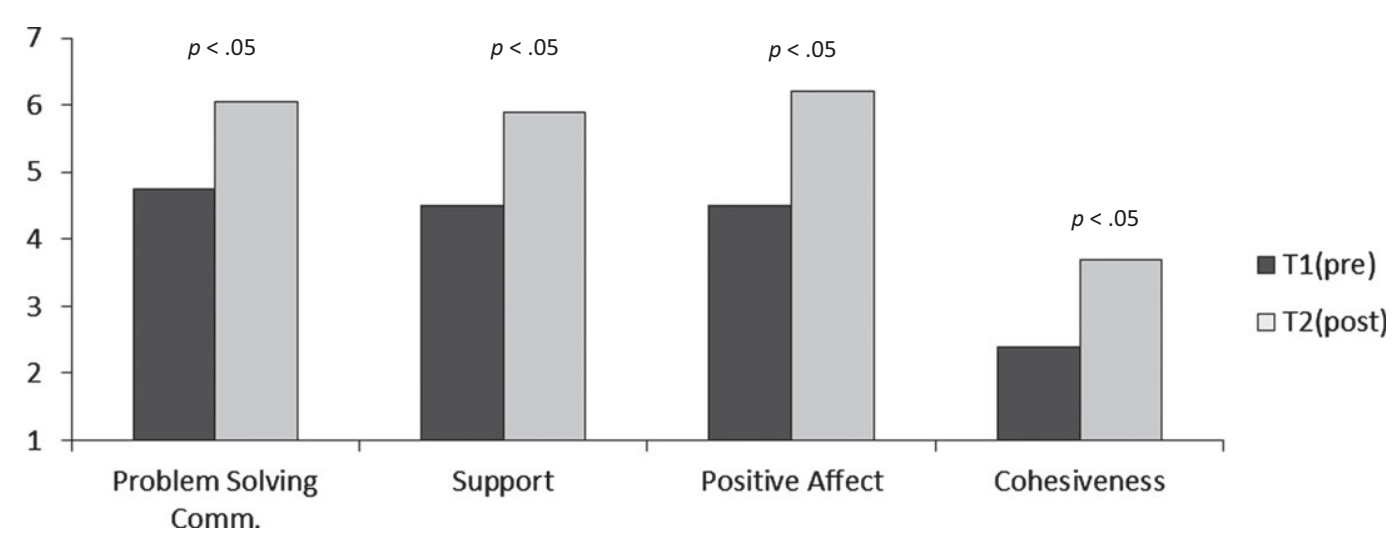

FIGURE 2. Mean scores for positive couple interaction variables. $p$ values represent the significance of paired $t$-tests comparing T1(PRE) with T2(POST).

PRE-POST decrease in Coerciveness $(r=.47, p<.05)$ and negatively related to the increase in Withdrawal $(r=-.61, p<.01)$.

\section{Did couples with greater cumulative family risk display more significant improvements?}

Regression analyses indicated no associations between extent of change on any of the coparent interaction indicators of interest and degree of cumulative family risk, after controlling for parents' SCID scores at point of intake. That is, data did not suggest that coparents who had experienced greater cumulative risk derived differential benefit from the intervention, at least not with regard to the particular coparent interaction processes examined. 


\section{Status of couples at the point of exit interviews}

At the point of study completion, only 1 of the 20 mother-father pairs (5\%), down from $35 \%$ at the point of intake, was judged to be showing High Conflict dynamics. Eight of the mother-father pairs (40\%), up from $20 \%$ at the point of intake, were judged to be showing either Disengaged (seven families) or Pursuit-Withdrawal dynamics (one family) during the discussions. The remaining 11 mother-father pairs (55\%, up from $45 \%$ at intake) were judged to be Harmonious on the basis of their interactions during the problem-solving task at intake. Of the six sets of coparents who at the point of intake had been engaging in destructive conflict (but who by study's end were no longer), three had moved to demonstrate Disengaged patterns during conflict; arguably, a safer situation for mother and baby, and the other three were now demonstrating Harmonious interactions. One family demonstrating a Pursuit-Withdrawal pattern at intake was also showing a Harmonious pattern by study's end. Arguably then, changes shown by seven families in which there had been signs of concern at intake were in positive directions.

Analyses suggested that 3 of the 20 coparenting teams had gravitated in a more negative direction - one moving from Disengaged dynamics at intake to High Conflict-Hostile dynamics at exit assessment (the topic breeding conflict during the postdiscussion was infidelity); and two moving from Harmonious to Disengaged (one) or Pursuit-Withdrawal (one) patterns. The remaining 10 families were evaluated as showing the same dynamic as that seen at intake - with seven Harmonious families remaining Harmonious, and three families showing Disengaged or Pursuit-Withdrawal patterns at intake showing similar dynamics at study's end. Overall, 14 of the 20 families demonstrated what can be considered beneficial outcomes, 3 did not improve, and 3 showed some signs of decline from their state at the point of intake.

\section{DISCUSSION}

Results from this study demonstrate significant declines in conflictual and destructive interpersonal dynamics and significant improvements in interparental rapport and problem-solving communication are shown by lower income unmarried African American coparents having a first child together following a targeted coparenting intervention delivered during the third trimester of the pregnancy. Effect sizes of the changes witnessed ranged from moderate to large, and were largely independent of the degree of fidelity to the intervention shown by the male-female Mentor team who delivered the intervention - though there was suggestive evidence that Mentor competence was associated with greater declines in negativity and conflict and in coercive exchanges between partners - and with smaller increases in withdrawal from PRE to POST intervention. These data are especially noteworthy in that the substantive changes documented occurred more than 3 months after completion of the intervention, indicating that there was staying power of lessons learned during the prenatal intervention.

Findings indicate the promise and value of meeting coparenting couples "where they are", and delivering an intervention that places the child, rather than the couple, as the focus of the intervention. The willingness of fathers, given an interested mother, to take part in the intervention is in stark contrast to recruitment rates of most larger MRE efforts reported in the literature, and the fact that 20 of 28 consented-in fathers-including 20 of 21 fathers who actually participated in FIOC Session 1 following the intake evaluation-completed the intervention through to the end indicates that both men and women found the intervention palatable and to their needs. These data are significant, and worthy of notice - when interventions speak to unmarried African American parents' 
concerns about the health and wellbeing of their shared child, parents respond with motivation, resolve, and enthusiasm.

We recognize that the size of the sample for this study and the absence of a control group preclude making claims about the FIOC intervention having caused the improvements in mother-father rapport, problem-solving, and communication reported. A larger randomized control trial will be needed to test causal hypotheses. We also acknowledge having followed best practice recommendations from the BSF and other MRE intervention studies enrolling low-income unmarried parents. These included implementation of the intervention at a trusted community human service agency, utilization of male and female recruiters and African American male-female Mentor teams well-known to the community and with substantial prior experience working with African American youth and new parents, flexibility in curricular delivery when family circumstances warranted (e.g., doubling up of sessions or conducting sessions as home visits in the late pregnancy for the final few sessions if mothers were put on bedrest), assistance with transportation, childcare, and provision of meals, and modest incentives for participation (each parent receiving a $\$ 50$ Babies-R-Us gift card for perfect session attendance). These accommodations likely also contributed to the strong recruitment and retention rates for the project, though probably not to the declines in destructive conflict or improvements in rapport, communication, and problem-solving.

It is also significant to note that participants in this project were broadly representative of participants in the national Fragile Families and Child Wellbeing study. All were lower income, fully half were not co-residential at PRE or at POST, and parents were less likely than those in either the BSF project or the FFCWB study to report optimism about the potential of future marriage. Yet the overwhelming majority recruited-in came, stayed, and appear to have derived benefit from participation. Unlike Epstein et al. (2015), we did not find differential benefits for higher risk couples, though in this project risk was defined as both inter- and extra-familial factors rather than as baseline couple conflict as was the case in the Epstein report.

In conclusion, the potential for future applications of a Focused Coparenting Consultation for families where marriage may be later in coming, or not in the cards at all, seems limitless. More research, employing randomized control trial methodology, will help to further elucidate the nature and scope of benefits to families who partake of the intervention. For the time being, it is safe to conclude that while more research is warranted, the metaphor of coparenting - a family strengths approach already copasetic within the African American community-may be a game-changer in efforts to strengthen higher risk families.

\section{REFERENCES}

Breitenstein, S. M., Fogg, L., Garvey, C., Hill, C., Resnick, B., \& Gross, D. (2010). Measuring implementation fidelity in a community-based parenting intervention. Nursing Research, 59(3), 158.

Devaney, B., \& Dion, R. (2010). 15-month impacts of Oklahoma's Family Expectations program. Princeton, NJ: Mathematica Policy Research.

Dion, R., Avellar, S., Zaveri, H., \& Hershey, A. (2006). Implementing healthy marriage programs for unmarried couples with children: Early lessons from the building strong families project, Mathematica Policy Research Reports, Mathematica Policy Research.

Edin, K., \& Reed, J. M. (2005). Why don't they just get married? Barriers to marriage among the disadvantaged. The Future of Children, 15(2), 118-137.

Epstein, K., Pruett, M. K., Cowan, P., Cowan, C., Pradhan, L., Mah, E. et al. (2015). More than one way to get there: Pathways of change in co-parenting conflict after a preventive intervention. Family Process, 54(4), 610618. doi:10.1111/famp.12138.

Huston, T. L., \& Melz, H. (2004). The case for (promoting) marriage: The devil is in the details. Journal of Marriage and Family, 66(4), 943-958. 
Karney, B. (2011). What's (not) wrong with low-income couples: Maintaining intimacy in more and less affluent marriage. Presented at the National Council on Family Relations Annual Conference, November 19, Orlando, Florida.

Knutson, N. M., Forgatch, M. S., Rains, L. A., \& Sigmarsdóttir, M. (2009). Fidelity of Implementation Rating System (FIMP): The manual for PMTO $^{\mathrm{TM}}$ (revised ed.). Eugene, OR: Implementation Sciences International.

Malik, N. M., \& Lindahl, K. M. (2004). System for coding interactions in dyads (SCID). In P. Kerig \& K. Lindahl (Eds.), Couple observational coding systems (pp. 173-188). Mahwah, NJ: Erlbaun.

McHale, J. (2007). Charting the bumpy road of coparenthood: Understanding the challenges of family life. Washington, DC: Zero to Three Press.

McHale, J., Gaskin-Butler, V., McKay, K., \& Gallardo, G. (2013). Figuring it out for the child initiative: Fostering coparenting in unmarried expectant African American parents. Zero to Three, 33(6), 17-22.

McHale, J., \& Irace, K. (2010). Focused Coparenting Consultation: Helping parents coordinate to support children. Independent Practitioner, 30, 164-170.

McHale, J. P., \& Lindahl, K. M. (2011). Coparenting: A conceptual and clinical examination of family systems. Washington, DC: American Psychological Association.

McHale, J., Waller, M. R., \& Pearson, J. (2012). Coparenting interventions for fragile families: What do we know and where do we need to go next? Family Process, 51(3), 284-306.

Minuchin, S. (1974). Families and family therapy. Cambridge, MA: Harvard University Press.

Minuchin, P., Colapinto, J., \& Minuchin, S. (2009). Working with families of the poor. New York: Guilford Press.

Owen, J., Quirk, K., Bergen, C., Inch, L. J., \& France, T. (2012). The effectiveness of PREP with lower-income racial/ethnic minority couples. Journal of Marital and Family Therapy, 38(s1), 296-307.

Salman-Engin, S., Begovic, E., Little, T., Knabel, S., Peterson, J., Gaskin-Butler, V. et al. (2013, March). Field implementation of "Figuring It Out for the Child": Early indicators of delivery fidelity. Paper presented at the meetings of the Southeastern Psychological Association, Atlanta, GA.

Wood, R. G., McConnell, S., Moore, Q., Clarkwest, A., \& Hsueh, J. (2010). Strengthening unmarried parents' relationships: Early impacts of Building Strong Families. Princeton, NJ: Mathematica Policy Research.

Wood, R. G., Moore, Q., \& Clarkwest, A. (2011). BSF's effects on couples who attended group relationship skills sessions: A special analysis of 15-month data. OPRE Report \#2011-17, Washington, DC: Office of Planning, Research, and Evaluation, Administration for Children and Families, U.S. Department of Health and Human Services. 